\title{
On the absence of global solutions for some $q$-difference inequalities
}

\author{
Hassen Aydi ${ }^{1 *}$, Mohamed Jleli ${ }^{2}$ and Bessem Samet ${ }^{2}$
}

\section{"Correspondence:}

hmaydi@iau.edu.sa

${ }^{1}$ Department of Mathematics,

College of Education in Jubail,

Imam Abdulrahman Bin Faisal

University, Jubail, Saudi Arabia

Full list of author information is

available at the end of the article

\begin{abstract}
In this paper, we obtain sufficient conditions for the nonexistence of global solutions for some classes of $q$-difference inequalities. Our approach is based on the weak formulation of the problem, a particular choice of the test function, and some q-integral inequalities.
\end{abstract}

MSC: 39A13; 35A23; 35B44

Keywords: Nonexistence; Global solution; q-difference inequalities

\section{Introduction}

In this paper, we obtain sufficient conditions for the nonexistence of global solutions for some classes of $q$-difference inequalities. First, we are concerned with the $q$-difference inequality

$$
\left(D_{q} y\right)(t) \geq|y(q t)|^{p}, \quad t>0,
$$

subject to the initial condition

$$
y(0)=y_{0},
$$

where $q \in(0,1), D_{q}$ is the $q$-derivative operator, $p>0$, and $y_{0}>0$.

Next, we study the system of $q$-difference inequalities

$$
\begin{cases}\left(D_{q} y\right)(t) \geq|z(q t)|^{p}, & t>0, \\ \left(D_{q} z\right)(t) \geq|y(q t)|^{m}, & t>0,\end{cases}
$$

subject to the initial condition

$$
y(0)=y_{0}, z(0)=z_{0},
$$

where $q \in(0,1), p>1, m>1$, and $y_{0}+z_{0}>0$.

In the limit case where $q \uparrow 1,(1)$ reduces to the ordinary differential inequality

$$
y^{\prime}(t) \geq|y(t)|^{p}, \quad t>0 .
$$

(c) The Author(s) 2019. This article is distributed under the terms of the Creative Commons Attribution 4.0 International License (http://creativecommons.org/licenses/by/4.0/), which permits unrestricted use, distribution, and reproduction in any medium, provided you give appropriate credit to the original author(s) and the source, provide a link to the Creative Commons license, and indicate if changes were made. 
It is well known that if $p>1$ and $y_{0}>0$, then problem (5)-(2) blows up in a finite time. Observe also that in the limit case where $q \uparrow 1$, (3) reduces to the system of ordinary differential inequalities

$$
\begin{cases}y^{\prime}(t) \geq|z(t)|^{p}, & t>0, \\ z^{\prime}(t) \geq|y(t)|^{m}, & t>0 .\end{cases}
$$

Note that if $p>1, m>1, y_{0}>0$, and $z_{0}>0$, then a blow-up situation takes place for problem (6)-(4) (see, e.g., [18]).

The study of sufficient conditions for the nonexistence of global solutions to differential equations or inequalities provides important information in theory as in applications. First, sufficient conditions for the absence of solutions provide necessary conditions for the existence of solutions. Second, useful information on limiting behaviors of many physical systems can be obtained via the nonexistence criteria. Indeed, having an information on the blowing-up of solutions can help in preventing accidents and malfunction in industry. It helps also in improving the performance of machines and extending their lifespan.

There are several works in the literature concerning the nonexistence of solutions for different classes of differential equations or inequalities involving nonstandard derivatives. In particular, the study of the absence of solutions for different types of fractional differential problems has received a great attention from many researchers. In this direction, we refer the reader to $[15,16,18-21]$ and the references therein. However, to the best of our knowledge, there are no investigations on the nonexistence of solutions in quantum calculus.

The $q$-difference calculus or quantum calculus is an old subject, which is rich in history and in applications. It was initiated by Jackson $[11,12]$ and developed by many researchers (see, e.g., $[1,6,8]$ ). We can find in the literature several papers dealing with the existence of solutions for different kinds of $q$-difference equations; see, for example, [3-5, 9, 10, 13, $17,24]$ and the references therein.

In this paper, we obtain sufficient criteria for the absence of global solutions to problems (1)-(2) and (3)-(4). The proofs are based on an extension of the test function method due to Mitidieri and Pohozaev [22] to quantum calculus.

The paper is organized as follows. In Sect. 2, we recall some basic concepts on $q$-calculus and present some properties and lemmas that will be used in the proofs of our results. Section 3 is devoted to study the nonexistence of global solutions for problem (1)-(2). In Sect. 4, we establish a nonexistence result for problem (3)-(4).

\section{Preliminaries on quantum calculus}

In this section, we recall some basic concepts on quantum calculus and provide some useful properties.

We denote by $\mathbb{N}$ the set of natural numbers and by $\mathbb{N}^{*}$ the set $\mathbb{N} \backslash\{0\}$. Here we follow [7, 23].

Let $q \in(0,1)$ be fixed. For $a \in \mathbb{R}$, we set

$$
[a]_{q}=\frac{1-q^{a}}{1-q} .
$$


The $q$-analog of the power $(a-b)^{N}$ is

$$
\begin{aligned}
& (a-b)^{(0)}=1, \\
& (a-b)^{(N)}=\prod_{i=0}^{N-1}\left(a-b q^{i}\right), \quad(a, b) \in \mathbb{R}^{2}, N \in \mathbb{N} .
\end{aligned}
$$

Let $f:[0, T] \rightarrow \mathbb{R}, T>0$, be a given function such that $f^{\prime}(t)$ exists in a neighborhood of $t=0$ and is continuous at $t=0$. The $q$-derivative of the function $f$ is defined by

$$
\left(D_{q} f\right)(t)=\frac{f(t)-f(q t)}{(1-q) t}, \quad 0<t \leq T
$$

and

$$
\left(D_{q} f\right)(0)=f^{\prime}(0)
$$

We easily see that $D_{q} f \in C([0, T] ; \mathbb{R})$.

Notice that if $f$ is differentiable, then

$$
\lim _{q \uparrow 1}\left(D_{q} f\right)(t)=f^{\prime}(t)
$$

The $q$-integral of the function $f$ is defined by

$$
\left(I_{q} f\right)(t):=\int_{0}^{t} f(s) d_{q} s=(1-q) t \sum_{n=0}^{\infty} q^{n} f\left(q^{n} t\right), \quad 0 \leq t \leq T
$$

provided that the sum converges absolutely. We say that $f$ is $q$-integrable on $[0, T]$ iff $\int_{0}^{t}|f(s)| d_{q} s<\infty$ for all $t \in[0, T]$. If $f$ is such that, for some $C>0$ and $\alpha>-1,|f(s)|<C s^{\alpha}$ in a right neighborhood of $s=0$, then $f$ is $q$-integrable (see [7]).

We can easily see that if $f$ is $q$-integrable on $[0, T]$, then

$$
\left|\int_{0}^{t} f(s) d_{q} s\right| \leq \int_{0}^{t}|f(s)| d_{q} s, \quad 0 \leq t \leq T .
$$

Moreover, if $f_{1}, f_{2}:[0, T] \rightarrow \mathbb{R}$ are two $q$-integrable functions on $[0, T], T>0$, then

$$
f_{1} \leq f_{2} \quad \Longrightarrow \quad \int_{0}^{T} f_{1}(s) d_{q} s \leq \int_{0}^{T} f_{2}(s) d_{q} s
$$

By [2], if $f$ is Riemann integrable on $[0, t]$, then

$$
\int_{0}^{t} f(t) d t=\lim _{q \uparrow 1}\left(I_{q} f\right)(t)
$$

Lemma 2.1 ([14]) Let $f \in C([0, T] ; \mathbb{R}), T>0$, be a continuous function such that $f^{\prime}(t)$ exists in a neighborhood of $t=0$ and is continuous at $t=0$. Then

$$
\left(D_{q}\left(I_{q} f\right)\right)(t)=f(t)
$$


and

$$
\left(I_{q}\left(D_{q} f\right)\right)(t)=f(t)-f(0) .
$$

Let $u(s)=\alpha s^{\beta}$, where $0 \leq s \leq t, \alpha>0$, and $\beta>0$. Then we have the change-of-variable formula (see [14])

$$
\int_{u(0)}^{u(t)} g(z) d_{q} z=\int_{0}^{t} g(u(s)) D_{q^{\frac{1}{\beta}}} u(s) d_{q^{\frac{1}{\beta}}} s,
$$

where $g:\left[0, \alpha t^{\beta}\right] \rightarrow \mathbb{R}$ is a $q$-integrable function on $\left[0, \alpha t^{\beta}\right]$.

Lemma 2.2 (see [7]) Let $N \in \mathbb{N}^{*}, T>0$, and $a, b, t \in \mathbb{R}$. Then

$$
D_{q}(a+b t)^{(N)}=[N]_{q} b(a+b q t)^{(N-1)}
$$

and

$$
\int_{0}^{T}(a+b t)^{(N-1)} d_{q} t=\frac{\left(a+\frac{b T}{q}\right)^{(N)}-a^{N}}{\frac{[N]_{q} b}{q}} .
$$

Next, we recall the following $q$-integration-by-parts rule.

Lemma 2.3 (see [14]) Let $f_{1}, f_{2} \in C([0, T] ; \mathbb{R}), T>0$, be two given functions whose ordinary derivatives exist in a neighborhood of $t=0$ and are continuous at $t=0$. Then

$$
\int_{0}^{T} f_{1}(s)\left(D_{q} f_{2}\right)(s) d_{q} s=\left[f_{1}(s) f_{2}(s)\right]_{s=0}^{T}-\int_{0}^{T} f_{2}(q s)\left(D_{q} f_{1}\right)(s) d_{q} s .
$$

\section{The absence of global solutions for problem (1)-(2)}

In this section, we establish the nonexistence of global solutions for problem (1)-(2). Our nonexistence result is stated in the following theorem.

Theorem 3.1 Let $p>1$ and $y_{0}>0$. Then problem (1)-(2) admits no global solutions in $C^{1}([0, \infty) ; \mathbb{R})$.

Proof We argue by contradiction. Suppose that problem (1)-(2) has a global solution $y \in$ $C^{1}([0, \infty) ; \mathbb{R})$. Let us take $N \in \mathbb{N}^{*}$ such that

$$
\int_{0}^{1} g_{N}(z) d_{q} z<\infty
$$

where

$$
g_{N}(z)=(1-z)^{\frac{-p}{p-1}}(1-z)^{(N)}, \quad 0 \leq z \leq 1 .
$$

For an arbitrary $T>0$, let us introduce the test function

$$
\varphi_{T}(t)=T^{-N}(T-t)^{(N)}, \quad 0 \leq t \leq T .
$$


Multiplying inequality (1) by $\varphi_{T}(t)$, using (7), and taking the $q$-integral over $[0, T]$, we obtain

$$
\int_{0}^{T}\left(D_{q} y\right)(t) \varphi_{T}(t) d_{q} t \geq \int_{0}^{T}|y(q t)|^{p} \varphi_{T}(t) d_{q} t
$$

Using a $q$-integration by parts (see Lemma 2.3), we obtain

$$
\int_{0}^{T}\left(D_{q} y\right)(t) \varphi_{T}(t) d_{q} t=\left[y(t) \varphi_{T}(t)\right]_{t=0}^{T}-\int_{0}^{T} y(q t)\left(D_{q} \varphi_{T}\right)(t) d_{q} t
$$

Using the initial condition (2) and the facts that $\varphi_{T}(T)=0$ and $\varphi_{T}(0)=1$, we get

$$
\int_{0}^{T}\left(D_{q} y\right)(t) \varphi_{T}(t) d_{q} t=-y_{0}-\int_{0}^{T} y(q t)\left(D_{q} \varphi_{T}\right)(t) d_{q} t
$$

Next, by (14) and (15) we obtain

$$
y_{0}+\int_{0}^{T}|y(q t)|^{p} \varphi_{T}(t) d_{q} t \leq \int_{0}^{T}|y(q t)|\left|\left(D_{q} \varphi_{T}\right)(t)\right| d_{q} t .
$$

On the other hand, we have

$$
\int_{0}^{T}|y(q t)|\left|\left(D_{q} \varphi_{T}\right)(t)\right| d_{q} t=\int_{0}^{T}\left(|y(q t)|\left(\varphi_{T}(t)\right)^{\frac{1}{p}}\right)\left(\left(\varphi_{T}(t)\right)^{\frac{-1}{p}}\left|\left(D_{q} \varphi_{T}\right)(t)\right|\right) d_{q} t .
$$

Using Young's inequality, we obtain

$$
\begin{aligned}
& \int_{0}^{T}|y(q t)|\left|\left(D_{q} \varphi_{T}\right)(t)\right| d_{q} t \\
& \quad \leq \frac{1}{p} \int_{0}^{T}|y(q t)|^{p} \varphi_{T}(t) d_{q} t+\frac{1}{p^{\prime}} \int_{0}^{T}\left(\varphi_{T}(t)\right)^{\frac{-p^{\prime}}{p}}\left|\left(D_{q} \varphi_{T}\right)(t)\right|^{p^{\prime}} d_{q} t
\end{aligned}
$$

where $\frac{1}{p}+\frac{1}{p^{\prime}}=1$. Therefore, by (16) and (17) we obtain

$$
y_{0}+\left(1-\frac{1}{p}\right) \int_{0}^{T}|y(q t)|^{p} \varphi_{T}(t) d_{q} t \leq \frac{1}{p^{\prime}} \int_{0}^{T}\left(\varphi_{T}(t)\right)^{\frac{-p^{\prime}}{p}}\left|\left(D_{q} \varphi_{T}\right)(t)\right|^{p^{\prime}} d_{q} t,
$$

which yields

$$
p^{\prime} y_{0} \leq \int_{0}^{T}\left(\varphi_{T}(t)\right)^{\frac{-p^{\prime}}{p}}\left|\left(D_{q} \varphi_{T}\right)(t)\right|^{p^{\prime}} d_{q} t
$$

Further, using (13) and (9), we obtain

$$
\begin{aligned}
& \left(\varphi_{T}(t)\right)^{\frac{-p^{\prime}}{p}}=T^{\frac{N}{p-1}}\left[(T-t)^{(N)}\right]^{\frac{-1}{p-1}}, \\
& \left|\left(D_{q} \varphi_{T}\right)(t)\right|^{p^{\prime}}=T^{\frac{-N p}{p-1}}\left([N]_{q}\right)^{\frac{p}{p-1}}\left[(T-q t)^{(N-1)}\right]^{\frac{p}{p-1}} .
\end{aligned}
$$


Hence

$$
\begin{aligned}
& \int_{0}^{T}\left(\varphi_{T}(t)\right)^{\frac{-p^{\prime}}{p}}\left|\left(D_{q} \varphi_{T}\right)(t)\right|^{p^{\prime}} d_{q} t \\
& \quad=T^{-N}\left([N]_{q}\right)^{\frac{p}{p-1}} \int_{0}^{T}\left[(T-t)^{(N)}\right]^{\frac{-1}{p-1}}\left[(T-q t)^{(N-1)}\right]^{\frac{p}{p-1}} d_{q} t
\end{aligned}
$$

Next, we have

$$
\begin{aligned}
{\left[(T-t)^{(N)}\right]^{\frac{-1}{p-1}}\left[(T-q t)^{(N-1)}\right]^{\frac{p}{p-1}} } \\
\quad=\frac{\prod_{i=0}^{N-2}\left(T-q^{i+1} t\right)^{\frac{p}{p-1}}}{\prod_{i=0}^{N-1}\left(T-q^{i} t\right)^{\frac{1}{p-1}}} \\
=\frac{1}{(T-t)^{\frac{1}{p-1}}} \frac{\prod_{i=1}^{N-1}\left(T-q^{i} t\right)^{\frac{p}{p-1}}}{\prod_{i=1}^{N-1}\left(T-q^{i} t\right)^{\frac{1}{p-1}}} \\
=\frac{1}{(T-t)^{\frac{1}{p-1}}} \prod_{i=1}^{N-1}\left(T-q^{i} t\right) \\
=\frac{T^{N-1}}{(T-t)^{\frac{1}{p-1}}} \prod_{i=1}^{N-1}\left(1-q^{i} \frac{t}{T}\right) \\
=\frac{T^{N-1}}{(T-t)^{\frac{1}{p-1}}\left(1-\frac{t}{T}\right)} \prod_{i=0}^{N-1}\left(1-q^{i} \frac{t}{T}\right)=\frac{T^{N-1-\frac{1}{p-1}}}{\left(1-\frac{t}{T}\right)^{\frac{p}{p-1}}}\left(1-\frac{t}{T}\right)^{(N)} .
\end{aligned}
$$

Therefore, from (19) we obtain

$$
\begin{aligned}
\int_{0}^{T}\left(\varphi_{T}(t)\right)^{\frac{-p^{\prime}}{p}}\left|\left(D_{q} \varphi_{T}\right)(t)\right|^{p^{\prime}} d_{q} t & =\left([N]_{q}\right)^{\frac{p}{p-1}} T^{\frac{-p}{p-1}} \int_{0}^{T}\left(1-\frac{s}{T}\right)^{\frac{-p}{p-1}}\left(1-\frac{s}{T}\right)^{(N)} d_{q} s \\
& =\left([N]_{q}\right)^{\frac{p}{p-1}} T^{\frac{-p}{p-1}+1} \int_{0}^{T} g_{N}(u(s)) D_{q} u(s) d_{q} s
\end{aligned}
$$

where $u(s)=\frac{s}{T}, 0 \leq s \leq T$, and $g$ is defined by (12). Using the change-of-variable formula (8), we obtain

$$
\int_{0}^{T}\left(\varphi_{T}(t)\right)^{\frac{-p^{\prime}}{p}}\left|\left(D_{q} \varphi_{T}\right)(t)\right|^{p^{\prime}} d_{q} t=\left([N]_{q}\right)^{\frac{p}{p-1}} T^{\frac{-1}{p-1}} \int_{0}^{1} g_{N}(z) d_{q} z
$$

Recall that from (11) we have $\int_{0}^{1} g_{N}(z) d_{q} z<\infty$. Further, from (18) we get

$$
p^{\prime} y_{0} \leq \frac{\left([N]_{q}\right)^{\frac{p}{p-1}}}{T^{\frac{1}{p-1}}} \int_{0}^{1} g_{N}(z) d_{q} z
$$

Since this inequality holds for every $T>0$, passing to the limit as $T \rightarrow \infty$, we obtain $y_{0} \leq 0$, which contradicts the fact that $y_{0}>0$. The obtained contradiction implies the nonexistence of a global solution to (1)-(2) for any $p>1$. 
4 The absence of global solutions for problem (3)-(4)

In this section, we derive a nonexistence result for problem (3)-(4).

Theorem 4.1 Let $p>1, m>1$, and $y_{0}+z_{0}>0$. Then problem (3)-(4) admits no global solutions in $C^{1}([0, \infty) ; \mathbb{R}) \times C^{1}([0, \infty) ; \mathbb{R})$.

Proof As previously, we argue by contradiction. Suppose that problem (3)-(4) has a global solution $(y, z) \in C^{1}([0, \infty) ; \mathbb{R}) \times C^{1}([0, \infty) ; \mathbb{R})$. Let us take $N \in \mathbb{N}^{*}$ such that

$$
\int_{0}^{1} g_{N}(z) d_{q} z<\infty \text { and } \int_{0}^{1} h_{N}(z) d_{q} z<\infty
$$

where $g_{N}$ is defined by (12), and

$$
h_{N}(z)=(1-z)^{\frac{-m}{m-1}}(1-z)^{(N)}, \quad 0 \leq z \leq 1 .
$$

For arbitrary $T>0$, let us consider the test function $\varphi_{T}$ defined by (13). Multiplying the first inequality in (3) by $\varphi_{T}(t)$, taking the $q$-integral over $[0, T]$, and using a $q$-integration by parts, we obtain

$$
y_{0}+\int_{0}^{T}|z(q t)|^{p} \varphi_{T}(t) d_{q} t \leq \int_{0}^{T}|y(q t)|\left|\left(D_{q} \varphi_{T}\right)(t)\right| d_{q} t .
$$

On the other hand, we have

$$
\int_{0}^{T}|y(q t)|\left|\left(D_{q} \varphi_{T}\right)(t)\right| d_{q} t=\int_{0}^{T}\left(|y(q t)|\left(\varphi_{T}(t)\right)^{\frac{1}{m}}\right)\left(\left(\varphi_{T}(t)\right)^{\frac{-1}{m}}\left|\left(D_{q} \varphi_{T}\right)(t)\right|\right) d_{q} t .
$$

Using Young's inequality, we obtain

$$
\begin{aligned}
& \int_{0}^{T}|y(q t)|\left|\left(D_{q} \varphi_{T}\right)(t)\right| d_{q} t \\
& \quad \leq \frac{1}{m} \int_{0}^{T}|y(q t)|^{m} \varphi_{T}(t) d_{q} t+\frac{1}{m^{\prime}} \int_{0}^{T}\left(\varphi_{T}(t)\right)^{\frac{-m^{\prime}}{m}}\left|\left(D_{q} \varphi_{T}\right)(t)\right|^{m^{\prime}} d_{q} t
\end{aligned}
$$

where $\frac{1}{m}+\frac{1}{m^{\prime}}=1$. Therefore, using (23) and (24), we obtain

$$
\begin{aligned}
y_{0} & +\int_{0}^{T}|z(q t)|^{p} \varphi_{T}(t) d_{q} t \\
& \leq \frac{1}{m} \int_{0}^{T}|y(q t)|^{m} \varphi_{T}(t) d_{q} t+\frac{1}{m^{\prime}} \int_{0}^{T}\left(\varphi_{T}(t)\right)^{\frac{-m^{\prime}}{m}}\left|\left(D_{q} \varphi_{T}\right)(t)\right|^{m^{\prime}} d_{q} t .
\end{aligned}
$$

Similarly, multiplying the second inequality in (3) by $\varphi_{T}(t)$, we obtain

$$
\begin{aligned}
z_{0} & +\int_{0}^{T}|y(q t)|^{m} \varphi_{T}(t) d_{q} t \\
& \leq \frac{1}{p} \int_{0}^{T}|z(q t)|^{p} \varphi_{T}(t) d_{q} t+\frac{1}{p^{\prime}} \int_{0}^{T}\left(\varphi_{T}(t)\right)^{\frac{-p^{\prime}}{p}}\left|\left(D_{q} \varphi_{T}\right)(t)\right|^{p^{\prime}} d_{q} t .
\end{aligned}
$$


Adding (25) to (26), we obtain

$$
\begin{aligned}
y_{0} & +z_{0}+\left(1-\frac{1}{m}\right) \int_{0}^{T}|y(q t)|^{m} \varphi_{T}(t) d_{q} t+\left(1-\frac{1}{p}\right) \int_{0}^{T}|z(q t)|^{p} \varphi_{T}(t) d_{q} t \\
& \leq \frac{1}{m^{\prime}} \int_{0}^{T}\left(\varphi_{T}(t)\right)^{\frac{-m^{\prime}}{m}}\left|\left(D_{q} \varphi_{T}\right)(t)\right|^{m^{\prime}} d_{q} t+\frac{1}{p^{\prime}} \int_{0}^{T}\left(\varphi_{T}(t)\right)^{\frac{-p^{\prime}}{p}}\left|\left(D_{q} \varphi_{T}\right)(t)\right|^{p^{\prime}} d_{q} t,
\end{aligned}
$$

which yields

$$
C_{m, p}\left(y_{0}+z_{0}\right) \leq \int_{0}^{T}\left(\varphi_{T}(t)\right)^{\frac{-m^{\prime}}{m}}\left|\left(D_{q} \varphi_{T}\right)(t)\right|^{m^{\prime}} d_{q} t+\int_{0}^{T}\left(\varphi_{T}(t)\right)^{\frac{-p^{\prime}}{p}}\left|\left(D_{q} \varphi_{T}\right)(t)\right|^{p^{\prime}} d_{q} t
$$

where $C_{m, p}=\min \left\{m^{\prime}, p^{\prime}\right\}$. On the other hand, from the proof of Theorem 3.1 we have

$$
\int_{0}^{T}\left(\varphi_{T}(t)\right)^{\frac{-p^{\prime}}{p}}\left|\left(D_{q} \varphi_{T}\right)(t)\right|^{p^{\prime}} d_{q} t=\left([N]_{q}\right)^{\frac{p}{p-1}} T^{\frac{-1}{p-1}} \int_{0}^{1} g_{N}(z) d_{q} z
$$

and

$$
\int_{0}^{T}\left(\varphi_{T}(t)\right)^{\frac{-m^{\prime}}{m}}\left|\left(D_{q} \varphi_{T}\right)(t)\right|^{m^{\prime}} d_{q} t=\left([N]_{q}\right)^{\frac{m}{m-1}} T^{\frac{-1}{m-1}} \int_{0}^{1} h_{N}(z) d_{q} z
$$

Therefore, from (27) we obtain

$$
C_{m, p}\left(y_{0}+z_{0}\right) \leq \frac{\left([N]_{q}\right)^{\frac{p}{p-1}}}{T^{\frac{1}{p-1}}} \int_{0}^{1} g_{N}(z) d_{q} z+\frac{\left([N]_{q}\right)^{\frac{m}{m-1}}}{T^{\frac{1}{m-1}}} \int_{0}^{1} h_{N}(z) d_{q} z .
$$

Passing to the limit as $T \rightarrow \infty$, we obtain a contradiction to

$$
y_{0}+z_{0}>0
$$

\section{Acknowledgements}

B. Samet extends his appreciation to Distinguished Scientist Fellowship Program (DSFP) at King Saud University (Saudi Arabia).

\section{Funding}

This work is supported by the Distinguished Scientist Fellowship Program (DSFP) at King Saud University.

\section{Competing interests}

The authors declare that they have no competing interests.

\section{Authors' contributions}

The authors declare that they carried out all the work in this manuscript and read and approved the final manuscript.

\section{Author details}

'Department of Mathematics, College of Education in Jubail, Imam Abdulrahman Bin Faisal University, Jubail, Saudi Arabia. ${ }^{2}$ Department of Mathematics, College of Science, King Saud University, Riyadh, Saudi Arabia.

\section{Publisher's Note}

Springer Nature remains neutral with regard to jurisdictional claims in published maps and institutional affiliations.

Received: 20 October 2018 Accepted: 22 January 2019 Published online: 30 January 2019 


\section{References}

1. Agarwal, R.P.: Certain fractional q-integrals and q-derivatives. Proc. Camb. Philos. Soc. 66, 365-370 (1969)

2. Agrawal, P.N., Kasana, H.S.: On simultaneous approximation by Szaz Mirakian operators. Bull. Inst. Math. Acad. Sin. 22, 181-188 (1994)

3. Ahmad, B., Alsaedi, A., Ntouyas, S.K.: A study of second-order q-difference equations with boundary conditions. Adv. Differ. Equ. 2012, 35, 1-10 (2012)

4. Ahmad, B., Nieto, J.J., Alsaedi, A., Hutami, H.A.L.: Existence of solutions for nonlinear fractional $q$-difference integral equations with two fractional orders and nonlocal four-point boundary conditions. J. Franklin Inst. 351, 2890-2909 (2014)

5. Ahmad, B., Ntouyas, S.: Boundary value problems for q-difference inclusion. Abstr. Appl. Anal. 2011, Article ID 292860 15 pages (2011)

6. Al-Salam, W.A.: Some fractional q-integrals and q-derivatives. Proc. Edinb. Math. Soc. 2, 135-140 (1966-1967)

7. De Sole, A., Kac, V.G.: On integral representations of q-gamma and q-beta functions. Atti Accad. Naz. Lincei, Rend. Lincei, Mat. Appl. 16, 11-29 (2005)

8. Ernst, T.: The history of $q$-calculus and a new method. UUDM Report 2000:16, Department of Mathematics, Uppsala University. 2000, ISSN: 1101-3591

9. Ferreira, R.A.C.: Positive solutions for a class of boundary value problems with fractional $q$-differences. Comput. Math. Appl. 61, 367-373 (2011)

10. Islam, M.N., Neugebauer, J.T.: Existence of periodic solutions for a quantum Volterra equation. Adv. Dyn. Syst. Appl. 11 67-80 (2016)

11. Jackson, F.H.: On q-functions and a certain difference operator. Trans. R. Soc. Edinb. 46, 253-281 (1908)

12. Jackson, F.H.: On q-definite integrals. Pure Appl. Math. Q. 41, 193-203 (1910)

13. Jia, L., Cheng, J., Feng, Z.: A q-analogue of Kummer's equation. Electron. J. Differ. Equ. 2017, 31, 1-20 (2017)

14. Kac, V.G., Cheung, P.: Quantum Calculus. Universitext. Springer, New York (2002)

15. Kassim, M., Furati, K.M., Tatar, N.E.: Non-existence for fractionally damped fractional differential problems. Acta Math. Sci. 37, 1-13 (2017)

16. Kassim, M.D., Furati, K.M., Tatar, N.E.: On a differential equation involving Hilfer-Hadamard fractional derivative. Abstr. Appl. Anal. 2012, Article ID 391062, 17 pages (2012)

17. Khodabakhshi, N., Vaezpour, S.M.: Existence and uniqueness of positive solution for a class of boundary value problems with fractional q-differences. J. Nonlinear Convex Anal. 16, 375-384 (2015)

18. Kirane, M., Malik, S.A.: The profile of blowing-up solutions to a nonlinear system of fractional differential equations. Nonlinear Anal. 73, 3723-3736 (2010)

19. Kirane, M., Medved, M., Tatar, N.E.: On the nonexistence of blowing-up solutions to a fractional functional differential equation. Georgian Math. J. 19, 127-144 (2012)

20. Kirane, M., Tatar, N.E.: Nonexistence of solutions to a hyperbolic equation with a time fractional damping. Z. Anal. Anwend. 25, 131-142 (2006)

21. Kirane, M., Tatar, N.E.: Absence of local and global solutions to an elliptic system with time-fractional dynamica boundary conditions. Sib. Math. J. 48, 477-488 (2007)

22. Mitidieri, E., Pohozaev, S.: A priori estimates and nonexistence of solutions of nonlinear partial differential equations and inequalities. Proc. Steklov Inst. Math. 234, 1-362 (2001)

23. Rajković, P.M., Marinković, S.D., Stanković, M.S.: Fractional integrals and derivatives in q-calculus. Appl. Anal. Discrete Math. 1, 311-323 (2007)

24. Yang, W.: Positive solutions for boundary value problems involving nonlinear fractional $q$-difference equations. Differ. Equ. Appl. 5, 205-219 (2013)

\section{Submit your manuscript to a SpringerOpen ${ }^{\circ}$ journal and benefit from:}

- Convenient online submission

- Rigorous peer review

- Open access: articles freely available online

- High visibility within the field

- Retaining the copyright to your article

Submit your next manuscript at $>$ springeropen.com 